\title{
La garance, la pomme de terre et le caillou : un naturaliste à histoire
}

\section{Georges Guille-Escuret}

\section{OpenEdition}

1 Journals

\section{Édition électronique}

URL : http://journals.openedition.org/jso/1353

DOI : $10.4000 /$ jso. 1353

ISSN : $1760-7256$

\section{Éditeur}

Société des océanistes

\section{Édition imprimée}

Date de publication : 1 décembre 2002

Pagination : 9-12

ISSN : 0300-953x

\section{Référence électronique}

Georges Guille-Escuret, « La garance, la pomme de terre et le caillou : un naturaliste à histoire », Journal de la Société des Océanistes [En ligne], 114-115 | Année 2002, mis en ligne le 26 mai 2008, consulté le 22 mars 2021. URL : http://journals.openedition.org/jso/1353 ; DOI : https://doi.org/ 10.4000/jso.1353

\section{(c) (i) ()}

Journal de la société des océanistes est mis à disposition selon les termes de la Licence Creative Commons Attribution - Pas d'Utilisation Commerciale - Pas de Modification 4.0 International. 


\title{
La garance, la pomme de terre et le caillou : un naturaliste à histoire
}

par

\author{
Georges GUILLE-ESCURET *
}

Cinq ans après sa disparition, il est toujours aussi difficile de parler de l'œuvre scientifique de Jacques Barrau. Ou, du moins, de distinguer l'importance du travail accompli de l'image personnelle du professeur, bien que la confusion risque de sacrifier l'analyse des apports proprement dits à l'évocation d'une aura ou d'un charisme. Sous l'effet d'un réflexe maladroit, devant des jeunes qui n'ont eu ni la chance de l'écouter ni le privilège d'entrer dans son bureaucapharnaüm de la rue Cuvier, nous demandons à l'histoire spectaculaire et exceptionnelle d'un homme de mettre en relief la valeur d'un chercheur dont, justement, la pensée ne s'est jamais voulue théâtrale et ne s'est surtout jamais imaginée en phare solitaire. Du lycéen résistant à l'universitaire inclassable, en passant par l'agronome anti-colonialiste, une certaine continuité dans l'intransigeance nous détourne de la cohérence intellectuelle. Un peu comme dans les textes signés par Georges Brassens : vingt ans ont passé, mais combien d'admirateurs pourraient entonner une de ses chansons sans que le souvenir d'un regard, d'une moustache et d'un sourire ne les oblige encore à recopier platement le tempo et les intonations de la version originelle? De même que, dans le cas du poète, la beauté intrinsèque des vers est absorbée par la réminiscence d'un timbre de voix, dans le cas du savant, de façon plus inattendue, le message reste imbibé par une individualité : l'abstraction ne s'est pas encore détachée du vécu.

En témoigne le fait que, dans cet hommage collectif rendu par les Océanistes à l'un des leurs, il ait été jugé souhaitable par les participants de consacrer quelques lignes à Barrau « lui-même » à travers son itinéraire scientifique. Ceci posé, et révérence gardée, un élève sur le point de se livrer à ce périlleux exercice peut se permettre d'ajouter que le patron lui-même a un tantinet compliqué les choses: entre autres, par de subtiles pudeurs intellectuelles et par de fines coquetteries de naturaliste bon teint, les unes et les autres passant d'autant plus inaperçues aux yeux de ses contemporains qu'elles ne découlaient pas simplement de son adhésion notoire au marxisme.

Pas question de reconstituer ici un puzzle en y espérant la cohérence globale d'une de ces esquisses que Barrau prisait fort : bornons-nous à fixer quelques repères en nous appuyant sur les mots qu'il employait pour se présenter.

Questions d'étiquette: entre histoire naturelle, biogéographie et écologie

Le premier de ces points d'ancrage tient bizarrement à la qualification disciplinaire de la recherche : Barrau se déclarait tout bonnement « naturaliste», avouant avec une feinte contrition une formation d'agronome. À la rigueur, il pouvait accepter l'étiquette d'ethnobotaniste qui ne l'engageait à rien sur un plan strictement théorique. Sauf erreur, il n'a pas fréquemment endossé la qualification d'ethnobiologiste, potentiellement plus ambiguë à cet égard, même quand son laboratoire l'a brandie dans son intitulé. Mais surtout, même oralement et à la cantonade, on ne l'entendait pas se targuer d'être « écologiste» (ou «écologue » après qu'une mouvance politique ait dérobé le premier terme),

* CNRS, UMR 8098 Techniques et Culture. 
ni même «biogéographe ». Or, personne n'aurait pu lui en contester le droit et, avec le recul, il apparaît que ce retrait ne provenait pas non plus d'une indifférence vis-à-vis des problèmes épistémologiques.

Au temps de ses études à Toulouse, il avait compté parmi les premiers privilégiés à recevoir un enseignement approfondi d'écologie authentique: à l'époque, en France, la phytologie monopolisait pratiquement le secteur (affirmant volontiers qu'une approche scientifique cessait d'être envisageable avec l'entrée en lice du monde animal dans les modèles) et Barrau a retenu au passage la récupération de notions sociologiques élémentaires par les spécialistes des communautés végétales. Plus tard, devenu professeur à Yale à un moment où cette université réunissait une pléthore de talents remarquables en anthropologie et en archéologie, il ne manqua pas de s'intéresser au "théâtre écologique " que, dans cette université, George Evelyn Hutchinson était en train de redéfinir, moyennant une clarification sous-jacente du support darwinien.

Barrau n'était en aucun cas un autodidacte de l'écologie qui aurait acquis son savoir «sur le tas » en confectionnant des herbiers. Il saisissait parfaitement l'originalité et la substance de la discipline. Plus encore, il en connaissait l'histoire théorique et politique depuis ses troubles balbutiements : l'œecologie d'Ernst Haeckel, bien sûr, mais également l'anthropogéographie de Friedrich Ratzel. Il manquait rarement l'occasion de rappeler ces références, avec les dangers attenants, quand ses collègues biologistes s'engageaient dans des raccourcis redoutables, ou retombaient sous la coupe de telle ou telle sirène réductionniste.

Disons-le abruptement: Barrau ne prenait pas très au sérieux les maîtres naïfs et pontifiants de cette écologie française qui attendit les années 80 pour accepter par force et du bout des lèvres d'inclure les actions humaines dans son champ de recherche. Sans effort sensible et dans l'intérêt évident du candidat, il fut capable de conserver un sourire quasi bouddhique au sein d'un jury de thèse, pendant que son voisin expliquait avec emphase le caractère fondamentalement adaptatif de l'ouverture d'un parapluie sous le mauvais temps.

Quant à la biogéographie, si elle le concernait d'aussi près et plus quotidiennement, comme support indispensable à cette reconstitution historique des liens entre hommes et nature à laquelle il était rivé, il n'a jamais voulu la confondre avec le lieu de synthèses plus élaborées (ou plus sophistiquées) qu'est l'écologie, et ceci même quand, de différentes sources et avec dif- férentes intentions, un vaste élan a voulu faire tomber les deux disciplines en synonymie.

Pour lui, ne pas mélanger les ambitions permettait de ne pas mélanger les enjeux, les devoirs et les responsabilités. Dans cet ordre d'idées, distinguer les cibles de l'ethnoscience de celles de l'ethno-écologie n'avait pas pour sens d'amoindrir l'une ou l'autre.

\section{Histoire naturelle et théories de la nature etlou de l'homme}

Barrau se trouvait alors inéluctablement en porte-à-faux. Sous l'étendard du naturalisme, il était censé prôner l'observation pour l'observation et dénigrer les efforts de l'interprétation, afin de se conformer à une image d'Épinal de la science qui montre des empiristes incultes et des théoriciens infirmes se tournant résolument le dos. Au lieu de cela, il a souvent servi de mémoire et de rempart contre les fausses candeurs de pensées savantes mimant l'impromptu. En outre, sa contribution à la recherche ethnologique a augmenté l'instabilité de sa position : pratiquement solidaire avec une ethnographie privilégiant le recueil méthodique des données, il ne laissait pas la critique d'une théorie glisser vers un dédain de principe à l'encontre de l'activité théorique.

Néanmoins, à quoi correspondait donc au départ ce souci de se dire naturaliste plutôt qu'écologue ou biogéographe. C'est ici que la pudeur et la coquetterie ont leur part : dans les sciences de la vie, l'histoire naturelle a en charge la mission encyclopédique, laquelle incarne indubitablement l'espérance classique la plus élevée en ce domaine. En maintes occasions, Barrau l'a d'ailleurs explicitement inscrite dans le sillage de la philosophie des Lumières et de l'humanisme.

L'écologie est une théorie de la nature issue du darwinisme, l'histoire naturelle est le champ colossal où cette théorie s'établit et qu'elle éclaire : à l'inverse de la vision plus récente des sciences humaines, le professeur du Museum avait conservé intacte la conviction que la théorie est un outil plus ou moins efficace pour acquérir et ordonner des informations, mais que la collecte de celles-ci demeure la tâche essentielle et prestigieuse. Chez les botanistes et les zoologistes, un diplôme suffit pour se déclarer écologue, mais, sauf à accepter le risque du ridicule, ne se proclame pas naturaliste qui veut: cette légitimité-là est autrement exigeante et la confrérie qu'elle engendre demande plus qu'un aval institutionnel. 
Barrau admirait et utilisait Darwin. Et il racontait volontiers comment André-Georges Haudricourt, dans le feu d'une conversation, était soudain entré dans une librairie du Quartier Latin pour en sortir deux minutes plus tard avec un volume qu'il lui jeta aussitôt dans les mains : Dialectique de la nature, d'Engels. Mais cela ne l'empêchait pas d'admirer sans retenue, dans de gros volumes $\mathrm{du} \mathrm{XIX}^{\mathrm{e}}$ siècle, les descriptions ébouriffantes de variétés végétales ou animales qui avaient été publiées par des auteurs d'autant plus minutieux qu'ils étaient résolument fixistes !

Quelques années après la guerre, et à la suite d'une démission houleuse de son poste d'agronome en Nouvelle-Calédonie, Barrau a sillonné pendant des mois l'Océanie comme un beatnik avant la lettre (le mot est de lui) et il a gardé de cette période un souvenir heureux, nostalgique peut-être, jusqu'à la fin de sa vie : la sensation de liberté qu'un jeune homme encore marqué par la déportation appréciait sans doute mieux qu'un autre était puissamment renforcée par l'impression que cette liberté n'était pas dénuée de sens et qu'elle accompagnait l'épanouissement d'une connaissance inattendue et enthousiasmante. L'universalité de la quête naturaliste est devenue en somme la réplique à l'ethnocentrisme autoritaire et arbitraire de l'agronomie occidentale du milieu du $\mathrm{xx}^{\mathrm{e}}$ siècle. Et le fruit de la résistance réveillée dans le fonctionnaire caldoche qui, avec effroi, s'était découvert, de par sa formation et de par son poste, le possible complice d'une oppression agissant par l'entremise d'une science hautaine.

Naturaliste, de ce point de vue, désignait plus que la fréquentation d'un district du savoir : la revendication d'une mission, voire d'un héritage. Une partie de la séduction exercée par Barrau sur certains anthropologues ne résiderait-elle pas dans le fait que cette revendication limpide, complètement et consciemment assumée par lui, n'a guère d'homologue dans la tradition des sciences sociales?

\section{Hypothèses et fructifications}

Chercher des histoires dans la nature, la nature dans une histoire, le passé dans un devenir, une promesse dans le passé, la trace des hommes dans la présence d'une plante, le rôle d'une plante dans un phénomène social, c'est finalement un questionnement unique : décomposable si l'on veut, mais non hiérarchisable. Barrau ne passait pas son temps à se demander si la botanique imprégnait l'ethnologie ou si l'ethnologie minait la botanique : dans la relation hommes/plantes, il ne voyait pas de sens unique et n'imaginait pas davantage que l'évolution technique en crée un. Parce que la nature est systématiquement historique et que l'histoire est obligatoirement naturelle, aucune solution de continuité ne saurait séparer, d'un côté, l'enquête sur les conditions de diffusion voici dix mille ans d'un foyer de domestication indo-malais et, de l'autre côté, une réflexion sur "plantes et hommes à l'aube du Xxi ${ }^{e}$ siècle $\gg{ }^{1}$.

Une distinction véritable émergera cependant pour l'historien des sciences, que l'on se bornera à mentionner brièvement ici : dans son analyse du berceau cultural asiatique et de sa diffusion vers l'Océanie, Jacques Barrau a si bien rendu hommage aux intuitions axiales de N. I. Vavilov, d'A.-G. Haudricourt et L. Hédin, et de C. O. Sauer qu'il a négligé de situer son apport personnel, comme si lui-même le considérait comme mineur. Or, le travail accompli dans un essai tel que «l'humide et le sec» n'est pas secondaire, loin de là : il est plutôt perpendiculaire. À travers des situations, des espèces et des lieux, il égrène ou décline des gammes d'interactions et des éventails logiques de transformations dont beaucoup n'avaient pas même été devinées avant lui, nous guidant dans une rigueur imperturbable pas même provocatrice, serait-on tenté de dire de l'écologie la plus biologique à l'ethnoscience la plus structurale. On ne sait qui eut le plus de raison de se montrer jaloux ${ }^{2}$.

L'écologie théorise des synthèses et modélise des approches, l'histoire naturelle échafaude «pour de vrai »: s'afficher naturaliste, c'était aussi refuser de choisir des types de contrastes à travailler, puis des enchaînements à privilégier. Rigueur de la comparaison, certes, mais dans une liberté jalousement préservée des azimuts de la comparaison. Osons une morale du côté de l'anthropologie, en pariant que Barrau ne la renierait pas : si l'ethnographe est bien le naturaliste des sciences sociales, il gagnera sûrement à se reconnaître lui-même comme théoricien compétent et à cantonner ses complexes d'infériorité dans des secteurs où ils se révèleront moins inhibiteurs et moins stérilisants.

1. Jacques Barrau, 1983. «Plantes et hommes au seuil du Xxi siècle », La Pensée, 234 : 65-74.

2. Jacques Barrau, 1965. «L'humide et le Sec. An Essay on Ethnobiological Adaptation to Contrastive Environments in the Indo-Pacific Area », Journal of the Polynesian Society, 74 : 329-346. Voir aussi : 1974. " L'Asie du Sud-Est, berceau cultural», Études Rurales, $\mathrm{n}^{\circ}$ 53-56 : 17-39. 


\section{Leçons de choses humaines}

Puisque, répétons-le, ce bref texte vient en complément de recherches authentiques pour compléter l'hommage par l'évocation d'un relief personnel, il est juste de lui donner la parole en citant la manière dont il racontait volontiers les racines de sa vocation aux étudiants :

«Du côté de ma famille paternelle, on cultivait autrefois la garance dans le Vaucluse, car elle permettait de donner une belle couleur rouge aux culottes de l'infanterie française. Hélas, au $\mathrm{XIX}^{\mathrm{e}}$ siècle, un chimiste allemand découvrit un moyen de fabriquer cette teinte artificiellement, et ceci en abaissant considérablement son prix de revient : il s'ensuivit une grave crise économique dans la région, avec des émigrations vers tous les azimuts, Océanie comprise.

La branche maternelle, pour sa part, était irlandaise, vivant tant bien que mal des pommes de terre jusqu'à ce que l'arrivée d'un champignon parasite n'en ruine soudain la production, provoquant comme chacun sait de grandes famines, des troubles politiques et une vague de départs forcés vers les contrées lointaines: l'Amérique, bien sûr, mais aussi les antipodes.
C'est ainsi que mon père a rencontré ma mère sur le « caillou », la Nouvelle-Calédonie : alors, vous comprenez, j'avais un compte à régler avec l'ethnobotanique! » ${ }^{3}$.

Il est douteux que ces avatars familiaux aient réellement hanté le fils de la bourgeoisie caldoche au point de déclencher une double passion adolescente pour l'histoire humaine des végétaux et l'histoire botanique des hommes. Néanmoins, cette façon de raccorder les univers, c'est un style très caractéristique.

Concluons avec une anecdote qui vaudra peut-être à l'auteur de ces lignes la visite d'un fantôme furibond. Un jour, des enfants océaniens ont amené au patron un arbuste qui le laissa perplexe. Ayant envoyé ce matériel à des spécialistes du groupe taxonomique pour détermination, il reçut rapidement une réponse excitée : l'arbre appartenait à une espèce inconnue, toute nouvelle! Les correspondants souhaitaient recevoir au plus vite d'autres échantillons de ce que l'on appellerait désormais Ficus barraui. Hélas, ce fut une erreur rédhibitoire que de lui avoir donné ce nom-là : on n'en a pas trouvé d'autre exemplaire. 